\title{
Das Interglazial von Fahrenkrug in Holstein
}

\author{
Ein Beitrag zur Frage des Buchenvorkommens im Jungpleistozän
}

\author{
Von F.-R. Averdieck, Kiel \\ Mit 3 Abbildungen und einer Tafel (I).
}

$\mathrm{Z}$ us a m me n f as ung. Die Neuuntersuchung des Interglazialvorkommens von Fahrenkrug bei Segeberg (Holstein) ergab ein saale/weichselinterglaziales Alter (Zone e bis i). Die Angabe von Rotbuche, Fagus silvatica L., muß auf eine Fehlbestimmung zurückgeführt werden.

Da bisher Rotbuchenfunde älteren Datums durch Nachuntersuchungen nicht bestätigt werden konnten, muß mit dem Fehlen von Fagus mindestens für das Jungpleistozän Nord- und Mitteleuropas gerechnet werden. Ausnahmen, die vermutlich in ein Würm-Interstadial zu stellen sind, sind aus dem Alpengebiet bekannt. Möglicherweise hängen hiermit fernverwehte Buchenpollen in einigen Fundplätzen des Brørup-Interstadials zusammen.

$\mathrm{Sum}$ mary. A new investigation of the interglacial of Fahrenkrug near Segeberg (Holstein) proved it to be of Saale/Weichsel interglacial age (Zone e-i according to Jessen \& Milthers 1928). The mentioning of beech (Fagus silvatica L.) apparently was a mistake in determination.

The older findings of beech could not be confirmed by later researches. It seems that Fagus was absent at least during the younger Pleistocene of Northern- and Middle-Europe. Exceptions are known from the Alps. Probably they come from an interstadial of the Würm Glaciation. Long range transported pollen grains of Fagus of some localities of the Brørup Interstadial are possibly connected herewith.

\section{Einleitung}

Das Dorf Fahrenkrug, ca. $2 \mathrm{~km}$ westlich von Segeberg, erregte die Aufmerksamkeit, als man im Jahre 1889 in geringer Tiefe auf ein „Braunkohlenlager“ gestoßen zu sein glaubte. Durch mehrere Bohrungen war unter etwa $6 \mathrm{~m}$ Deckgebirge eine Mächtigkeit von 2 bis $3 \mathrm{~m}$ bei einer Ausdehnung von ca. 45 ha erschlossen worden. Der Versuch, dieses Lager wirtschaftlich auszubeuten, wurde nach Anlage eines tief en Grabens im angeblichen Zentrum des Feldes aufgegeben. Schon vorher war ein Schachtbau heruntergetrieben worden, durch den es C. A. WEBER ermöglicht wurde, den Aufbau des Flözes zu studieren. In seiner 1893 veröffentlichten Abhandlung „Über die diluviale Flora von Fahrenkrug in Holstein" gab WEBER ein umfassendes Bild über die Verlandungsfolge und den fossilen Floreninhalt des Aufschlusses. Das von ihm vorgefundene Profil lautet in abgekürzter Form:

$\begin{array}{lc}\text { Geschiebelehm } & 4,5 \mathrm{~m} \\ \text { Waldtorf } & 0,75 \\ \text { Sphagnumtorf } & 0,30 \\ \text { Hypnumtorf } & 0,05-0,12 \\ \text { leberartiger Torf (Mudde) } & 0,25 \\ \text { schwarzgrauer, pflanzenführender, sandiger Schluff } & \\ \quad \text { mit Ubbergängen nach oben und unten } & 0,15 \\ \text { toniger Feinsand mit humosen Beimengungen } & 1,0 \\ \text { harter Ton (Geschiebelehm?) } & \text { ca. } 2,0\end{array}$

In dem Einfallen des Flözes und seiner Schichtungen sah Weber eine spätere Störung, die er, wie die offensichtliche Abtragung an seiner Oberfläche, auf Gletscherschub zurückführte. Wesentlich war, daß WeBer in dem Aufbau der Schichten und ihrem Fossilinhalt ein a tochthones Torflager pleistozänen Alters erkannte.

Die in sorgfältigen Analysen gewonnene floristische Ausbeute enthielt innerhalb der Waldflora die Rotbuche. Unter den Wasserpflanzenfunden sind die im Holozän bei uns fehlende Nymphaeacee Cratopleura und die ausgestorbene Najadacee Paradoxocarpus carinatus NeHRING hervorzuheben. 


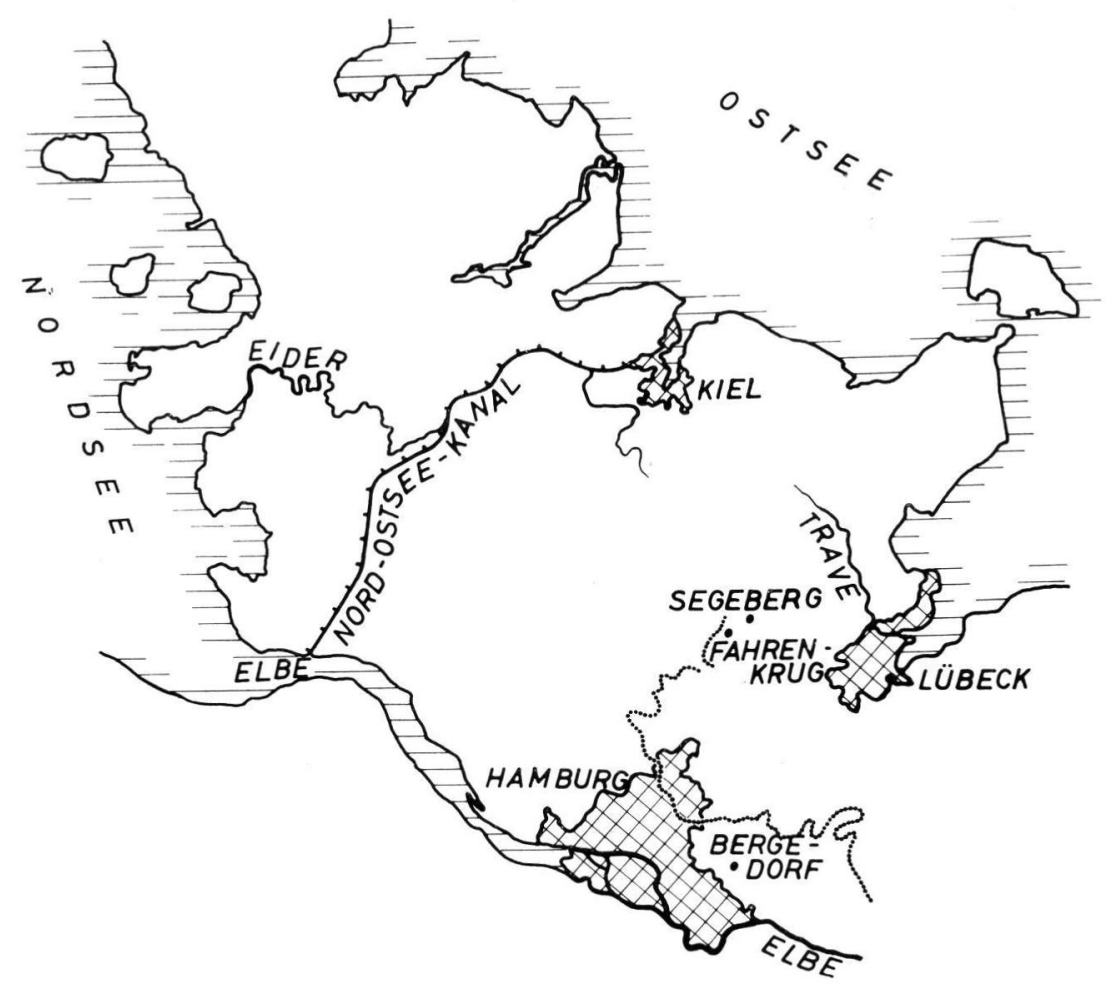

Abb. 1. Ubersichtsskizze. Punktierte Linie = Grenze der Weichsel-Vereisung.

Als die Fortschritte späterer Jahre eine genauere Gliederung des Pleistozäns erlaubten, wurde die Stellung des Fahrenkruger Interglazials zweifelhaft. Das von WEBER angegebene Vorkommen der Rotbuche, Fagus silvatica L., paßte nicht zu einem jungpleistozänen Alter, wie es nach der Lagerung unter geringmächtigem Geschiebelehm der letzten Vereisung zu erwarten war. So sahen manche Bearbeiter (z. B. P. Range 1945) in dem Torflager eine vom Eis verschleppte ältere Scholle, welche Deutung sich jedoch schlecht mit Webers Grabungsbefund vertrug. Das Interglazial von Fahrenkrug hat aufgrund seines Floreninhaltes in der Interglazial-Literatur seither stets eine Rolle gespielt, wobei die Buchenfunde einerseits zur Deutung solcher Funde anderer Gegenden herangezogen wurden, andererseits dagegen zur Begründung der Fragwürdigkeit der Altersstellung dienten (Jessen \& Milthers 1928, Reich 1953, Woldstedt 1955).

\section{Die neuen Bohrungen}

Trotz der fundamentalen Bedeutung, die dem Rotbuchen-Nachweis innerhalb der Vegetationsentwicklung in einem nordwestdeutschen Interglazial zukommt, war merkwürdigerweise bisher noch keine pollenanalytische Bearbeitung vorgenommen worden.

Im Jahre 1960 konnten vom Geologischen Landesamt Schleswig-Holstein einige Bohrungen auf dem von WEBER beschriebenen Gelände vorgenommen werden (Abb. 2). Außer Bohrung 4 traf keine andere Bohrung nach Durchstoßen der Geschiebelehmdecke auf humose Schichten, auch nicht die nahe der vermutlichen WEBER'schen Untersuchungsstelle gelegene Bohrung 3. Wenn auch noch Bohrungen im Süden ausstehen, so scheint doch die Vorstellung von einer Ausdehnung des Vorkommens auf 45 ha, dessen Zentrum bei Bohrung 3 hätte liegen sollen, übertrieben. 


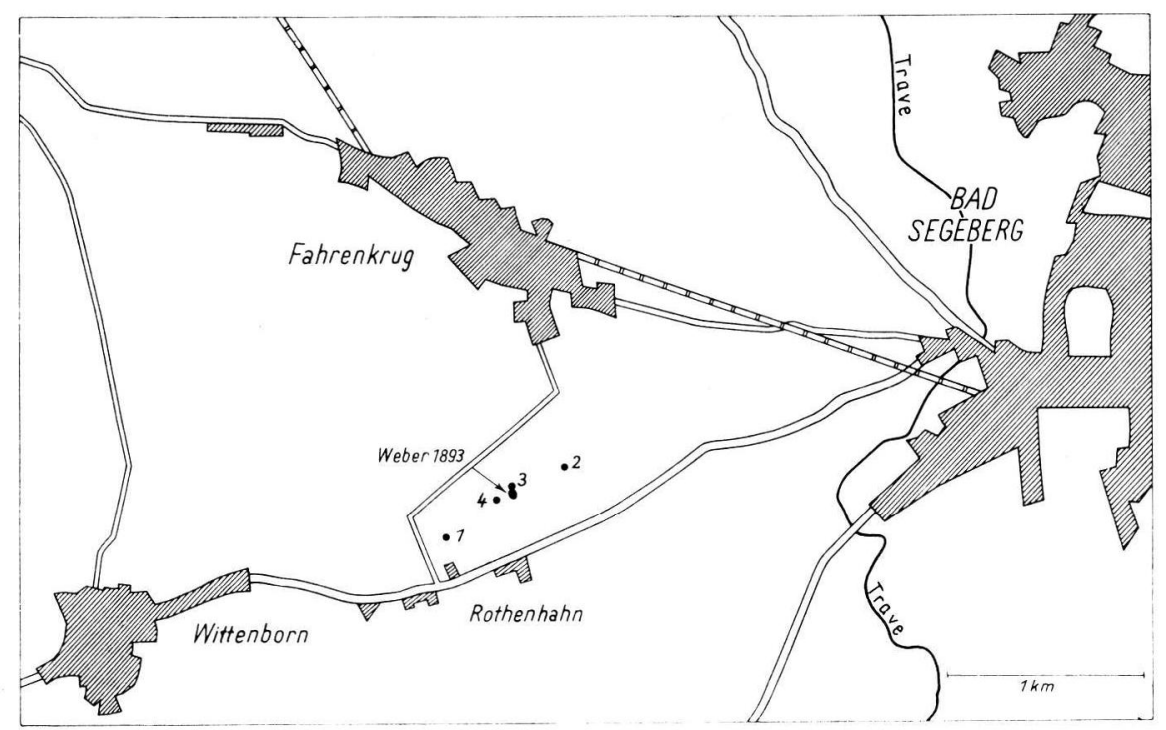

Abb. 2. Lageplan der Bohrungen 1960.

Die Bohrergebnisse lauten im einzelnen:

Bohrung Fahrenkrug 1

$$
\begin{array}{ccl}
0 & 4,30 \mathrm{~m} & \text { Geschiebelehm } \\
\text { - } 4,52 & \text { Mittelsand, grau } \\
\text { - } 5,40 & \text { Geschiebelehm } \\
\text { - } 6,08 & \text { Mittelsand mit Rostbändern } \\
\text { - } 6,15 & \text { Ton, sandig, braun } \\
\text { - }>10,00 & \text { Feinsand } \\
\hline
\end{array}
$$

Bohrung Fahrenkrug 2

$$
\begin{array}{ccl}
0- & 1,60 \mathrm{~m} & \text { Geschiebelehm } \\
- & 2,10 & \text { Mittelsand, z. T. lehmig } \\
- & 3,00 & \text { Sand, kiesig-steinig, rostbraun } \\
- & 4,00 & \text { Mittelsand, weiß-grau } \\
-\quad 7,40 & \text { Geschiebemergel, grau } \\
->8,40 & \text { Fein-Mittelsand, grau, gelegentlich humose Flitter }
\end{array}
$$

Bohrung Fahrenkrug 3

$$
\begin{array}{cll}
0- & 2,00 \mathrm{~m} & \text { Geschiebelehm } \\
\text { - } 3,75 & \text { Geschiebemergel } \\
\text { - } 3,90 & \text { Ton, schluffig, kalkhaltig (Beckenton?) } \\
\text { - } & 8,85 & \text { Geschiebelehm } \\
\text { - }>10,00 & \text { Geschiebemergel } \\
\hline
\end{array}
$$

Bohrung Fahrenkrug 4

$$
\begin{aligned}
& 0-4,30 \mathrm{~m} \quad \text { Geschiebelehm } \\
& \text { - 4,95 Geschiebemergel } \\
& \text { - 5,00 Geschiebemergel, humusstreifig } \\
& \text { - } 5,30 \quad \text { G y t t ja, schwarzbraun } \\
& \text { - 5,47 Feinsand, schluffig, tonig } \\
& \text { - 7,65 Torf } \\
& \text { - } 8,00 \quad \text { Gyttja } \\
& \text { - } 9,00 \quad \text { B e cke n t on, schluffig, feinsandig } \\
& \text { - 9,40 Feinsand mit Tonlagen, B e cke n a bs a t } \mathrm{z} \\
& \text { - 14,00 Mittelsand mit Tonlagen und Feuersteinen } \\
& \text { - 20,75 Mittel- und Grobsand, gelegentlich Tonlagen und Steine } \\
& \text { - 24,00 Mittel- und Grobsand, einzelne Steine, kalkhaltig }
\end{aligned}
$$


Der organische Anteil des Profiles 4 bot, da eine Kernbohrung ungestörter Proben vorgenommen worden war, gute Möglichkeiten für die Pollenanalyse. In beschränktem Maße konnten auch Makrofossilien gewonnen werden, die in der nachfolgenden Profilbeschreibung mit aufgeführt werden.

Profil Fahr
$5,00-5,30 \mathrm{~m}$
$5,30-5,47$
$5,47-5,55 \mathrm{~m}$
$5,55-5,70$
$5,70-5,85$

$5,85-6,00$
$6,00-6,50$
$6,50-7,50$
$7,50-7,65$

krug, Bohrung 4

G y t ja, tonig-schluffig, mit Tonstreifen. Zahlreiche Steinkerne von Potamogeton natans, einer von Sparganium spec., 1 Carex Sektio Eucarex-Innenfrucht, einige unbestimmte Samen (cf. Cruciferae). Einzelne CyperaceenEpidermen. Vereinzelt Drepanocladus fluitans 1 ). Pediastrum selten.

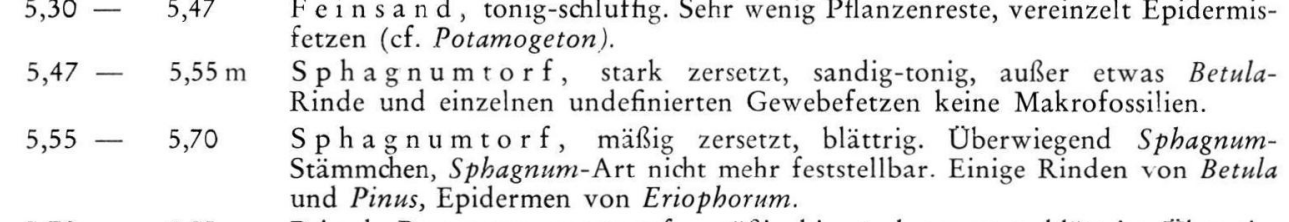
$\mathrm{R}$ i ed-Braunmoostorf, mäßig bis stark zersetzt, blättrig. Uberwiegend Stämmchen von Braunmoosen. Mehrere Früchte von Carex Sektio Eucarex, einige Carex-Schlauchreste und Epidermen von Cyperaceen. Vereinzelt Rinde von Betula und Holzfasern von Pinus.

$\mathrm{K}$ i ef e r n-Fichte $\mathrm{n}-\mathrm{Br}$ u chwald t orf, bröckelig, überwiegend Coniferen-Rinden und Holz von Picex, 1 Steinkern von Rubus fruticosus s.l., 1 unbestimmte Frucht. Einige Sklerotien von Coenococcum geophilum.

Fichten-Bruchwaldtorf mit Feinsandlinsen, bröckelig. Uberwiegend Coniferen-Rinden und Holzgrus von Picea. Zahlreiche Steinkerne von Rubus, meist fruticosus s. l., Steinkern von Sambucus racemosus. Vereinzelte Sklerotien von Coenococcum geophilum.

Erlen-Bruchwald torf, bröckelig. Uberwiegend Holzgrus und Rinden von Alnus. Vereinzelt Coniferen-Rinde. Früchte von Alnus und Betula. Men yanthes-B raunmoostorf, blättrig. Überwiegend Cratoneurum filicinum, vereinzelt Sphagnum spec. Zablreiche Samen und Epidermen von Menyanthes. Einige Steinkerne von Potamogeton natans und Innenfrüchte von Carex Sektio Vignea. Einige Fruchtwandstücke von Tilia cf. platyphyllos. Einige unbestimmte Früchte oder Samen. Gelegentlich Farnannuli.

7,65 - 7,85 m Gyttja, tonig, geschichtet, mit blättrigen Braunmoostorflagen. Früchte und Blattreste von Ceratophyllum submersum regelmäßig.

Vereinzelte Früchte bzw. Samen von Potamogeton cf. gramineus, Nymphaea alba (cf. microsperma), Urtica dioeca und Lycopus europaeus. Einige unbestimmte Früchte. Braunmoos- und Sphagnum-Blättchen.

$7,85-8,00 \quad$ Feindetritus-Gyt t ja, tonig, weich, wenig Makrofossilien. Mehrere Früchte und Blattreste von Ceratophyllum submersum. Vereinzelte Früchte bzw. Samen von Salix, Batrachium, Umbelliferen (cf. Oenanthe), Potamogeton natans und Carex Sektio Vignea.

Knospenschuppen und Holzreste, wenigstens z. T. von Quercus.

8,00 - 9,00 To n, feinsandstreifig, mit geringem Kies- und Grobsandanteil. Pflanzenreste selten, meist Holzkohle, 1 Potamogeton-Steinkern.

Leider kam es infolge des Bohrverfahrens mit einem $1 \mathrm{~m}$ langen Kernrohr zu einem Kernverlust zwischen 6,70 und $7,40 \mathrm{~m}$. Hieran war zu einem Teil der Holzreichtum der Bruchtorfschicht schuld, zum Teil die Stauchung durch das Bohrohr. Gerade aber wegen der Stauchung ist der tatsächliche Kernverlust wesentlich geringer zu veranschlagen, als er bei der nicht entzerrt wiedergegebenen Teufenskala des Pollendiagramms erscheint.

Während die Tiefenlage und die Schichten im Hangenden und Liegenden gute Übereinstimmung mit WEBER's Befunden zeigen, ergeben sich beim Vergleich der Torfschichten Abweichungen. In Abb. 3 sind die vereinfachten Profile mit den horizontweise eingetragenen stratigraphisch wichtigen, vorherrschenden Baumarten nebeneinandergestellt. Es zeigt sich hierbei, daß dem Profil von Weber der obere Teil fehlt, der im Profil 4 von der

1) Für die Bestimmung der Moose bin ich Herrn Dr. F. Koppe, Bielefeld, zu Dank verpflichtet. 


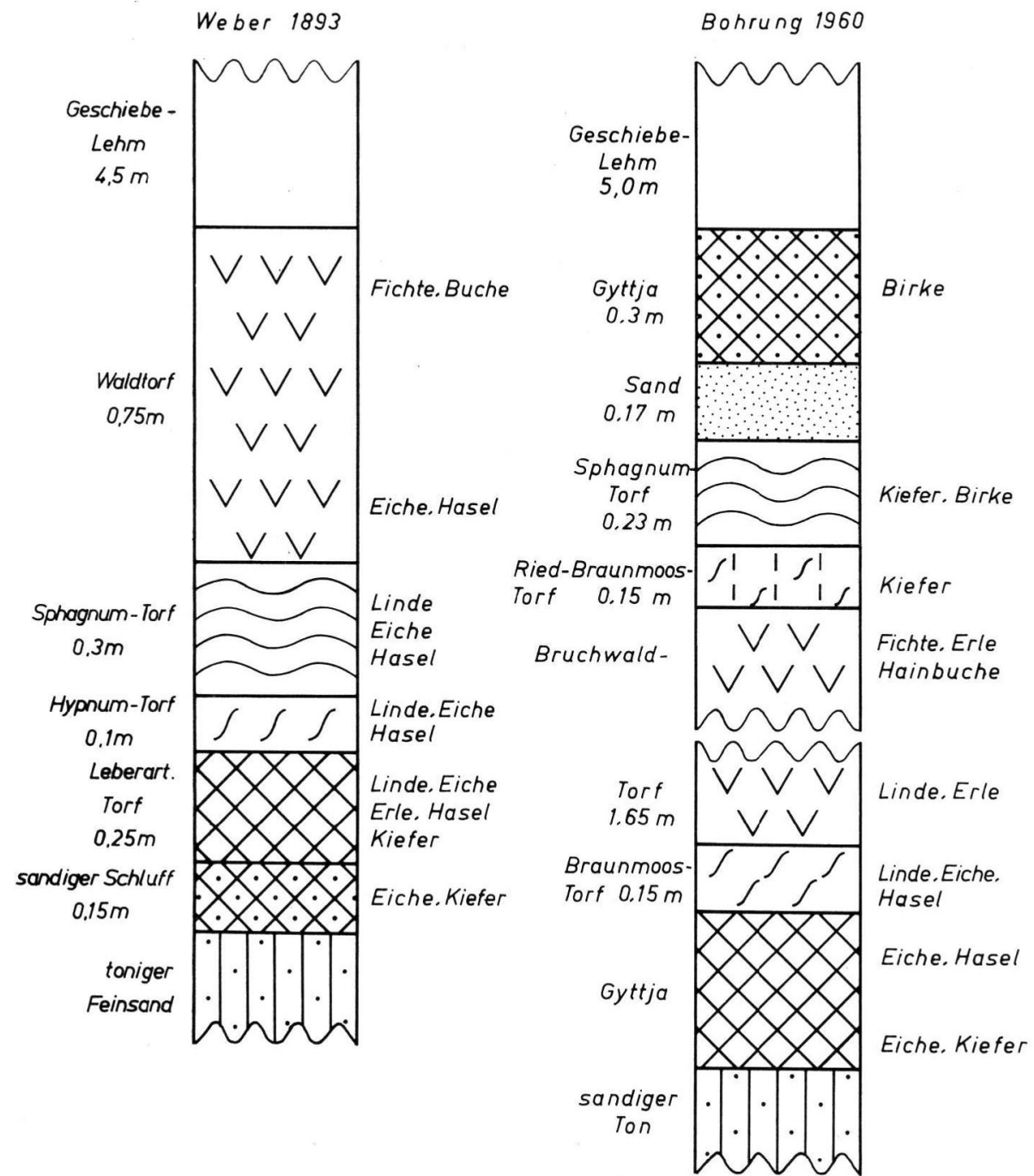

Abb. 3. Vergieich der Profile Grabung W'eber 1893 und Bohrung 4/1960.

Fichtenzeit an aufwärts erfaßt wurde. Dieser Umstand ist aber für die Prüfung der Buchenfrage besonders wichtig, da in dem neuen Profil der fragliche Abschnitt unbedingt enthalten sein muß.

\section{Pollendiagramm und Vegetationsentwicklung}

Zur Pollenanalyse wurden Proben nach Möglichkeit in 5-cm-Abständen entnommen. Die Aufbereitung erfolgte mittels Kalilauge und Acetolyse der bergfeuchten Substanz, bei Proben 5,30, 8,00 und $8,40 \mathrm{~m}$ außerdem mittels Flußsäure. Ausgezählt wurde auf mindestens 200 Baumpollen ohne Hasel, in Ausnahmefällen auf 100 Baumpollen.

Die Pollenerhaltung war im allgemeinen zufriedenstellend, in einigen Partien der Bruchwaldtorfe jedoch sehr schlecht, worunter besonders die Identifizierbarkeit des Eichenpollens litt. Die Darstellung erfolgte wegen des wechselhaften Einflusses des jeweiligen Moorbestandes in der herkömmlichen Form, bei der die Summe der Waldbäume unter Ausschluß von Corylus die Bezugsgrundlage für alle übrigen Pollentypen abgibt. 
Das Pollendiagramm (Tafel I) zeigt durchaus die bekannte Abfolge des Eeminterglazials. Die unterste Gyttja gehört bereits dem eichenbeherrschten Abschnitt e nach der Gliederung von Jessen \& Milthers an, in dem die Kiefer noch eine größere Rolle spielt, die Linde aber fehlt. Es darf als sicher gelten, daß das WEBER'sche Profil zur gleichen Zeit begonnen hat.

Der Abschnitt f zeigt den charakteristischen, gegenüber dem Postglazial verspätet erscheinenden Haselgipfel mit Werten bis über $400 \%$ der Baumpollensumme. Ebenso typisch sind die hohen UUlmen- und Lindenwerte, die eine beherrschende Rolle dieser Bäume im Waldbild nach Rückgang der Eiche andeuten. Wie im entsprechenden Abschnitt des Postglazials fand sich der Pollen von Hedera fast regelmäßig. Unter den Lindenpollen tritt der Platyphyllos-Typ häufig hervor, was sich mit den Fruchtfunden in diesem wie in dem Weber'schen Profil deckt. Tilia platyphyllos ist, wie auch aus den Untersuchungen von Jessen \& Milthers hervorgeht, im Eeeminterglazial gegensätzlich zum Postglazial im Norden sehr verbreitet gewesen.

In der Zone $\mathrm{g}$ ist das Carpinus-Maximum zwar deutlich, aber gegenüber anderen Eemdiagrammen nur dürftig ausgebildet. Außer einer möglichen selektiven Pollenzersetzung ist insbesondere die Überrepräsentanz der örtlichen Bruchtorfbildner Erle und Fichte dafür verantwortlich zu machen. Mit diesem Abschnitt verschwindet die Linde, während Eiche, Ulme und Hasel mit niedrigen Werten und später nur noch sporadisch in den nächsten hineinragen.

Zone h steht unter der Vorherrschaft von Picea, die gegen Ende bei ansteigender Pinus über 40\% erreicht. Das Pollenbild wird zu Beginn der Zone stark von Alnus beherrscht, obschon sich von der Erle keinerlei Großreste im Torf fanden. Das bedeutendste Kriterium dieser Zone ist das regelmäßige Auftreten von Abies-Pollen, der maximal 2\% erreicht. Da dieser Pollen nicht gerade weit streut (Finbas 1949), kann man annehmen, daß die Umgebung von Segeberg noch innerhalb der eemzeitlichen Ausbreitung der Tanne gelegen hat. Ihr Pollen wurde auch noch bei Schleswige ${ }^{2}$ ), nicht mehr aber in Dänemark gefunden (vgl. auch die Studie von SELle 1960).

An der oberen Grenze tritt Pinus bereits als Bestandteil des Bruchmoores in Erscheinung. Da hier auch die Salix-Kurve sowie ein Anstieg der Gramineenpollen einsetzen, ist die Pinus-Herrschaft wohl nicht nur auf örtliche Moorbesiedelung zurückzuführen. Vielmehr ist es im Zuge der Klimaverschlechterung zur Ausbildung eines ausgeprägten Kiefernwaldgürtels gekommen, der in gewissem Grade auch einen lichtbedürftigen Unterwuchs gestattete.

Der restliche Teil der Torfschicht, die in ihrer Zusammensetzung die gleiche Vernässung andeutet, wie sie von ANDERSEN (1961) in Dänemark und von ZaGwiJn (1961) in Holland festgestellt wurde, gehört der Zone i an. Dem Verschwinden der thermophilen Bäume zufolge wird diese Zone neuerdings bereits in das frühe Weichselglazial gestellt. Anzeichen für eine offene Vegetation sind hier aber noch recht spärlich. Die Zunahme des Nichtbaumpollens hat ihre Ursache in erster Linie in dem mooreigenen Bewuchs, zunächst durch Cyperaceen, später durch Calluna und Empetrum. Eine Beteiligung von Betula nana ist nicht nachzuweisen. Indessen spricht der Birkenanstieg wie auch die Zunahme der Mannigfaltigkeit der Krautpollen für eine gewisse Lichtung. Die Beteiligung von Empetrum hermaphroditum (im Pollendiagramm zusammengefaßt und mit Vaccinium- und Ledum-Typen in der Kurve der „anderen Ericales"), vielleicht auch das Auftreten von Rubus chamaemorus, läßt auf ein Heranrücken der Waldgrenze schließen.

Der obere stark zersetzte Sphagnum-Torf $(5,47-5,55)$ weist mit seinem Mineralreichtum auf periglaziale Störungen hin, wenn auch sichere Anzeichen mangels einwandfrei erkennbarer Sekundärpollen fehlen. Letztere sind in dem schluffigen Feinsand im Han-

2) Nach freundl. mündl. Mitteilung von Herrn Dr. Kolumbe, Hamburg-Altona. 


\begin{tabular}{|c|c|c|c|c|c|c|c|c|c|c|c|c|c|c|c|c|c|c|c|c|c|c|c|c|c|c|}
\hline \multicolumn{2}{|l|}{ Botrychium } & I & & & I & & 11 & 1 & & & 1 & & & 11 & & & & 11 & 11 & & & 11 & & & 11 & \\
\hline \multicolumn{2}{|l|}{ Polypodium } & 1 & 11 & 1 & 11 & 11 & 11 & 1 & 11 & 1 & 1 & & 1 & $1 \%$ & 1 & & & 11 & $1|1|$ & 11 & & 11 & & & 111 & \\
\hline \multicolumn{2}{|l|}{ Pteridium } & 11 & & 1 & $1:$ & 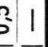 & 11 & 1 & 11 & 1 & 1 & & । & 11 & 11 & & & & & 11 & & 11 & 1 & & 11 & \\
\hline \multicolumn{2}{|c|}{ Varia indeterminata } & \pm 2 & & & & s. & $\hat{0}=$ & $=\left[\begin{array}{ll}n \\
0 \\
0\end{array}\right.$ & & & a & & ? & 11 & 11 & & & & & & $\frac{-1}{4}$ & 120 & 1 & & $=0$ & \\
\hline \multicolumn{2}{|l|}{ Potamogeton } & 11 & & 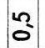 & & $1 \mid 1$ & 11 & I I & I 1 & I & 1 & I & 1 & 11 & & & & & ||$l \mid$ & & 1 & & 1 & & $=1$ & \\
\hline \multicolumn{2}{|l|}{$\begin{array}{l}\text { Myriophyllum } \\
\text { alterniflorum }\end{array}$} & & 11 & 1 & & 1 & 111 & 1 & & 1 & I & & 1 & 111 & & & 1 & 11 & 1 & 11 & & & 1 & & 11 & \\
\hline \multicolumn{2}{|l|}{ Batrachium } & 11 & 1 I & 1 & & 1 & 1 & 1 & & I & 1 & & 1 & I 1 & & 1 & 1 & 1 & 1 & 11 & & & 1 & & 1 & \\
\hline \multicolumn{2}{|l|}{ Nymphaea } & 11 & 11 & 1 & & 1 & 1 & 1 & & 1 & 1 & & 1 & 11 & & 1 & 1 & 1 & 1 & 11 & & 11 & - & & 11 & \\
\hline \multicolumn{2}{|l|}{ Nuphar } & 11 & 11 & 1 & & 1 & 1 & 1 & & I & 1 & & 1 & 11 & I & 1 & 1 & 1 & 1 & 11 & & $\because$ & $\tilde{0}$ & & 11 & \\
\hline \multicolumn{2}{|l|}{ Alisma } & 11 & 1 & 1 & & $-\infty$ & 11 & 1 & 11 & 1 & 1 & & 1 & 11 & 1. & 1 & 1 & 11 & 1 & 11 & & 11 & -1 & 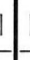 & 10 & \\
\hline \multicolumn{2}{|l|}{ Typha latifolia } & 11 & 11 & 1 & & 1 & 1 & 1 & 11 & 1 & 1 & & 1 & 11 & 1 & o & 1 & 11 & 1 & $1=$ & & $=-$ & $z$ & & 11 & 1 \\
\hline \multicolumn{2}{|l|}{ Sparganium-Typ } & 112 & 2 & 1 & 11 & 1 & 1 & 1 & 11 & 1 & 1 & & 1 & 11 & E & I & & $\because$ & 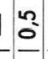 & 10 & & $\stackrel{n}{=}$ & . & & $\underset{n}{2}$ & \\
\hline \multicolumn{2}{|l|}{ Menyanthes } & 11 & 11 & 1 & & 1 & 11 & 1 & 11 & I & 1 & 11 & I & 11 & 1 & 11 & 1 & 11 & & 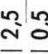 & 1 & 11 & 1 & & 11 & 1 \\
\hline \multicolumn{2}{|c|}{$\begin{array}{l}\text { Compos. tubuliflorae, } \\
\text { außer Artemisia }\end{array}$} & $1=$ & $-\overline{1}$ & & & $5-$ & 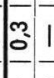 & 1 & 1 & I & I & 1 & 1 & 1 & 1 & I I & 1 & 11 & 1 & I 1 & 1 & 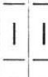 & 1 & I & $I^{2}$ & \\
\hline \multicolumn{2}{|c|}{ Compos.-liguliflorae } & 11 & 11 & 1 & 11 & $1 \%$ & 11 & 1 & 11 & 1 & I & & ? & 11 & 1 & I I & 1 & 11 & 1 & 11 & 1 & 11 & 1 & & 11 & 1 \\
\hline \multicolumn{2}{|l|}{ Galium-Typ } & 11 & 1.1 & 1 & 11 & 1. & $1 \hat{0}$ & 1 & 11 & $=$ & 1 & 11 & 1 & 11 & & 11 & 1 & 11 & 11 & 11 & & 11 & -1 & & 11 & It \\
\hline \multicolumn{2}{|l|}{ Labiatae } & 11 & 11 & 1 & 11 & 1 & 11 & 1 & 11 & I & 1 & & 1 & 11 & 1 & 11 & 1 & 11 & . & 1 & 1 & 11 & 1. & & $\stackrel{n}{0} 1$ & 1 \\
\hline \multicolumn{2}{|l|}{ Armeria } & 11 & 11 & 1 & 11 & 1 & 11 & 1 & 11 & I & 1 & 11 & 1 & 11 & 1 & 1 & 1 & 11 & 1 & 11 & 1 & 11 & -1 & & 11 & \\
\hline Lysim & & 11 & 1 - 1 & 1 & 11 & $1-1$ & 1 & 1 I & I I & 1 & 1 & 11 & 1 & $\begin{array}{lll}0 & 1 \\
0 & 1\end{array}$ & 1 & 11 & 1 & 11 & 1 & 11 & 1 & 11 & -1 & & 11 & I \\
\hline nbe & & 11 & $1 \%$ & $=$ & $\begin{array}{ll}n \\
0 \\
0\end{array}$ & $=8$ & 11 & 11 & $\begin{array}{l}0 \\
0 \\
0\end{array}$ & 1 & 1 & 11 & 1 & 11 & 1 & $1 \vec{c}$ & 1 & 11 & 1 & I & & 11 & 1 & & $\simeq \pm$ & 1 \\
\hline $\begin{array}{l}\text { Helianthemum } \\
\text { Typ }\end{array}$ & & 11 & 11 & 1 & 11 & 11 & 1 & 11 & 11 & 1 & 1 & 11 & 1 & 11 & & 1 & 1 & 11 & & I 1 & & 11 & I & & 11 & $\approx$ \\
\hline Epilobium & & 1 & 1 I & 1 & 11 & 1 & 1 & 11 & 11 & 1 & 1 & 11 & 1 & 11 & & 11 & 1 & 11 & 1 & $\stackrel{10}{\circ} 1$ & -1 & 11 & -1. & & 11 & 1 \\
\hline Crucif & & & 5 & 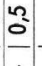 & $1 \stackrel{2}{0}$ & $s-$ & 1 & 1 & 11 & $\cong$ & & -1 & 1 & 11 & 1 & 11 & 1 & 11 & 1 & 11 & 1 & 11 & 1 & & 11 & I \\
\hline de & & 110 & 2. & 1 & 11 & 1 & 11 & $1 ?$ & I 1 & 1 & I & I 1 & 1 & 11 & 1 & 1 & 1 & 11 & 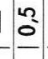 & 11 & 1 & 11 & 1 & & 11 & 1 \\
\hline Rubus chamaen & & 11 & 1 & 1. & 11 & 1 & $=0$ & 1 & 11 & 1 & 1 & I 1 & 1 & 11 & 1 & 1 & 1 & 11 & 1 & I I & 1 & 11 & 1. & 1 & 11 & I \\
\hline Comarum- & & I & $1 \%$ & 1 & 11 & 1 & 11 & 11. & 11 & $\cong$ & & $\simeq 1$ & 1 & $1=$ & I & 1 & 1 & 1 & 1 & I I & 1 & 11 & 1 & 1 & 11 & - \\
\hline Filipendula & & 12 & 21 & 1 & 11 & 1 & 10 & $=?$ & 11 & 1 & 1 & 1 I & 1 & 11 & 1 & 1 & 1 & 1 & 11 & 1 & 1 & 1 & -1 & 1 & 11 & | \\
\hline dere Ranuncul & & 11 & $1 \stackrel{0}{0}$ & 1 & $1 \div$ & $=1$ & 1 & 11 & 10 & & 1 & 11 & 1 & 11 & 1 & I & E & 1 & 1 & 11 & 1 & 1 & 1 & 1 & 11 & | \\
\hline Ialic & & o & $\frac{1}{5}$ & I & 11 & 1 & 11 & 1 I & I I & 정 & 1 & I I & 1 & 11 & 1 & 11 & 1 & 1 & 1 & 11 & 1 & 1 & 1 & 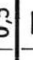 & 11 & I \\
\hline Caryophyllaceae & & ? & 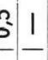 & 1 & 1 & 1 & 110 & $\begin{array}{lll}? & 1 \\
2 & \end{array}$ & 11 & I & 1 & 11 & 1 & 11 & 1 & 1 & 1 & 1 & 1 & III & 1 & 1 & 1 & | & 11 & 1 \\
\hline Chenopodiaceae & & 11 & 1 I & 1 & 1 & 1 & 11 & 11 & 11 & I & 1 & I I & 1 & 11 & 1 & 1 & I & 11 & -1 & 1 & 1 & 11 & 1 & 1 & 11 & 7 \\
\hline sto & & 11 & 11 & 1 & 114 & 8 点 & 11 & 11 & 1 I & $\frac{1}{2}$ & 1 & 11 & 1 & 11 & 1 & 11 & I & 11 & 1 & 11 & & 11 & 1 & & 11 & 1 \\
\hline Rumex acefosa- & & 19 & $\begin{array}{llll}0 & & 1 \\
& & \end{array}$ & & -1 & & 11 & 1 & I I & 0 & ñ & ?2. & 1 & II I & 1 & I & 1 & 11 & 1 & \begin{tabular}{lll} 
& \\
\hdashline & 1 &
\end{tabular} & & 11 & 1 & & 11 & \\
\hline $\begin{array}{l}\text { Ericales (außer } \\
\text { und Empetrum }\end{array}$ & & & $=\mid$ & 1 & 11 & 5 & & & & & 1 & ?. & 1 & 11 & 1 & & 1 & 11 & 1 & & & 11 & 1 & & 11 & \\
\hline 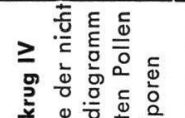 & 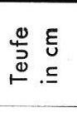 & & & & & & & & & & & & & & & & & & & & & & & & œ & \\
\hline 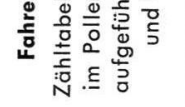 & $N$ & & & & & & & & & & & & & - & & or & & & & & 4 & & & & 0 & \\
\hline
\end{tabular}


genden $(5,30-5,47 \mathrm{~m})$ durch mehr oder weniger korrodierte Pollenexinen von Alnus, Pinus, Betula, Tilia und Corylus nebst Farn- und Sphagnum-Sporen reichlich vertreten. Eine quantitative Erfassung erübrigt sich hier.

Die oberste Gyttja scheint bereits einem Interstadial anzugehören mit ähnlichen klimatischen Bedingungen wie in der Zone i. Wie weit die geringen Pollenwerte von Picea, Alnus und wärmeliebenden Holzarten, zum größten Teil sicher auch von Pinus, auf Ferneinflug oder auf Sekundärverlagerung zurückzuführen sind, läßt sich angesichts der mineralischen Beimengung der Gyttja nicht entscheiden. Da die Fortführung dieser Schicht offensichtlich gekappt worden ist, wie humose Streifen an der Basis des hangenden Geschiebelehms zeigen, läßt sich auch eine sichere Bestimmung des Interstadials nicht durchführen. Sehr wahrscheinlich deutet aber der Pinus-Anstieg in Verbindung mit dem Auftreten einzelner kleiner Picea-Pollen vom Omorika-Typ und höheren Werten von Frangula alnus in der obersten Probe auf eine Zugehörigkeit zum Brørup-Interstadial hin. Eine Frangula-Häufung am Kiefernanstieg findet sich in ähnlicher Weise in Profilen vom Brørup-Hotel-Moor (ANDERsen 1961), von Odderade (noch unveröffentlicht), von Harksheide und von Lüneburg (beide HALLIK 1952, 1955), die sämtlich dem Brørup-Interstadial entstammen.

Während die Vegetationsentwicklung des Interglazials von der Wärmezeit bis in die Zeiten des heranrückenden Eises gut zu verfolgen ist, liegen aus dem vorhergehenden Teil keine Ablagerungen vor. Von dem sandstreifigen Ton im Liegenden der Torfe und Gyttjen wurden Proben in $10 \mathrm{~cm}$ Abständen untersucht. Bis auf die im Diagramm verzeichnete Probe $8,40 \mathrm{~m}$ war jedoch die Pollenmenge für eine rationelle Bearbeitung zu gering. Außerdem ließ sich keine Änderung der Pollenverteilung innerhalb des Schichtkomolexes feststellen. Als mit Sicherheit umgelagert ließen sich unregelmäßig vorkommende Sporen und Pollen des Tertiärs und Altpleistozäns, Pollen thermophiler Laubbäume und meist ziemlich korrodierte Pollen von Erle, Fichte und Tanne ausmachen. Ebenso mag seinem Erhaltungszustand nach ein größerer Teil der Pinus-Pollen und ein geringerer der BetulaPollen, die in annähernd gleichem Verhältnis den Baumpollengehalt beherrschen, zu beurteilen sein. Unter den merkwürdigerweise nicht reichlichen Krautpollen waren Pollen bzw. Sporen von Hippophaë, Helianthemum, Artemisia und Selaginella selaginoides vertreten. Die Birkenpollen wiesen einen auffallend hohen Anteil kleiner zarter Typen auf, wie er für Betula nana bezeichnend ist. Alles in allem kann also das Sediment als spätwartheeiszeitlicher Be cke nton angesprochen werden.

\section{Zur Frage der Fagus-Funde}

Wie eingangs erwähnt, fällt das Fahrenkruger Interglazial in seinem Florengehalt keineswegs aus dem Rahmen unserer bisherigen Erfahrungen über das Eeminterglazial heraus. Von der Gattung $F a g u s$ w urde bei dieser Untersuchung ke ine $S p u r$ gefunden. Da es nach den vorhergehenden Ausführungen als sicher gelten kann, daß beide Profile dem gleichen Torflager entstammen, daß das neue Profil Ablagerungen eines größeren Zeitraumes als das alte umfaßt, und daß die Flora nicht von der benachbarter Vorkommen abweicht, muß mit einer Fehlbestimmung WEBERs gerechnet werden. Womit möglicherweise eine Verwechslung erfolgt ist, muß völlig offen bleiben. Ein dem Fagus-Pollen im schlechten Erhaltungszustande nicht unähnlicher großer vierporfaltiger Rumex-Pollen wurde nur einmal im Fichtenbruchwaldtorf in 6,10 m Teufe beobachtet. Noch weniger erklärbar bleibt die Bestimmung des Holzes. Es ließe sich schließlich noch denken, daß infolge irgendeiner Störung auf die wärmezeitlichen Torfschichten die Ablagerungen eines buchenreichen Interstadials, wie es z. B. von FIRBAS (1958) bei Wasserburg am Inn beschrieben wurde, gelangt wären. Gegen solche Konstruktion spricht jedoch sowohl das Torfprofil selbst als auch die Tatsache, daß bei keinem der gut unter- 
suchten und vollständig entwickelten Interstadialvorkommen von den Niederlanden bis Dänemark jemals die Buche in Erscheinung trat.

Bestätigt werden konnte indessen WEBER's Befund, daß das Fahrenkruger Interglazial autochthon gewachsene Ablagerungen darstellt.

JESSEN \& MiLthers (1928) erwähnen neben Fahrenkrug als weiteres „Interglazial unsicheren Alters" ein Vorkommen bei Bergedorf östlich Hamburg. Auch hier war von Weber (in Koert 1912) innerhalb einer gemäßigten Flora Pollen von Fagus angegeben worden. Die Untersuchung eines Profiles, das in nächster Nachbarschaft und mit sehr ähnlichen stratigraphischen Verhältnissen angetroffen wurde, ergab jedoch ein junges postglaziales Alter (Averdieck 1951, Profil „St").

In der Literatur findet man noch eine Reihe weiterer Angaben über jungpleistozäne Fagus-Vorkommen im nördlichen Mitteleuropa (vgl. z. B. Woldstedt 1955). In mehreren Fällen sind Neuuntersuchungen vorgenommen worden, und es hat sich herausgestellt, daß entweder die Fagus-Angaben auf Fehlbestimmungen zurückzuführen sind (z. B. im bekannten Eeminterglazial von Szelag $=$ Schilling bei Posen, nach SRodon 1956), oder daß infolge Vernachlässigung stratigraphisch wichtiger Pollentypen die Datierung zu jung ausfiel (z. B. beim buchenführenden Pliozäntorf von Eichenberg, nach CHanda 1960). In manchen Fällen, in denen an der Zuverlässigkeit der Bestimmung nicht gezweifelt zu werden braucht, sind Buchenpollenfunde an derart starken Mineralgehalt des Sedimentes gebunden, daß eine sekundäre Allochthonie der Pollen wahrscheinlich ist. Dies gilt auch für ältere Interglazialvorkommen, abgesehen von der Tegelenwarmzeit (z. B. Bilshausen, LüTTIG \& REIN 1954). Obschon Nachuntersuchungen weiterer zweifelhafter Interglaziale wünschenswert wären, ist hinsichtlich der Buchenfrage im norddeutschen Raum mit Überraschungen kaum zu rechnen.

Weniger klar dürften einstweilen aber die Verhältnisse im Gebiet der nördlichen Voralpen liegen, wo nach FIRBAS (1958) in Ablagerungen wahrscheinlich würminterstadialen Alters gelegentlich eine auffällig hohe Fagus-Beteiligung zu finden ist. Die von H. REICH (1953) eingehend bearbeiteten eeminterglazialen Schieferkohlen von Großweil-Ohlstadt und Pfefferbichl, die offensichtlich auch noch ein Aquivalent des Brørup-Interstadials einschließen und bezüglich ihrer Vegetationsfolge im Einklang mit den norddeutschen Verhältnissen stehen, sind buchenfrei.

In diesem Zusammenhange sei noch eine Beobachtung am Rande erwähnt. Ein (vereinzeltes) Fagus-Pollenkorn fand der Verfasser kürzlich im brørup-interstadialen Sphagnumtorf von Odderade in Dithmarschen. In gleicher Torfart und Lagerung erwähnt H. REICH (1953) einen, von ihr allerdings ungewürdigten, Fagus-Pollen im Profil Großweil III. Beide Funde stammen gleichermaßen aus Ablagerungen, deren Zugehörigkeit zum Brørup-Interstadial unter anderem auch durch den Gehalt an Picea omorikoides angezeigt wird. Da angesichts der Torfart eine Sekundärverlagerung unwahrscheinlich ist, müssen die Buchenpollen durch Ferneinwehung hineingeraten sein. Von vereinzelten Buchenpollen, die auffallenderweise in jüngeren, kiefern- und fichtenbeherrschten Interglazialabschnitten der Schweizer Voralpen vorkommen, berichtet Lüdr (1953). Die Möglichkeit, daß zwischen diesen fernverwehten Buchenpollen einerseits und einem buchenführenden Interstadial andererseits, wie etwa dem von Wasserburg, Beziehungen bestehen könnten, sollte die Aufmerksamkeit bei künftigen Untersuchungen auf diese Frage lenken.

\section{Literaturverzeichnis}

Andersen, S. Th.: Vegetation and its Environment in Denmark in the Early Weichselian Glacial (Last Glacial) - Danmarks Geol. Unders. II R. 75, 175 S., Kopenhagen 1961.

Averdieck, F.-R.: Pollenanalytische Untersuchungen zur Vegetationsgeschichte im Osten Hamburgs. - Mitt. geogr. Ges. Hamburg 53, S. 161-176, Hamburg 1958. 
ChANDA, S.: Spättertiäre und interglaziale, pollenführende Ablagerungen im Leinetal nahe Göttingen und im sïdwestlichen Harzvorland (Untereichsfeld). - Naturwissenschaften 47, S. 19-20, Berlin 1960.

Firbas, F.: Spät- und nacheiszeitliche Waldgeschichte Mitteleuropas nördlich der Alpen, Bd. 1, 480 S., Jena 1949. - - Uber das Fagus-Vorkommen von Wasserburg am Inn (Oberbayern). - Veröff. geobot. Inst. Rübel 33, S. 81-90, Zürich 1958.

Hallik, R.: Ein „Weichsel-Frühglazial“-Profil in Lüneburg. - Eiszeitalter u. Gegenwart 2, S. 168172, Ớhringen/Württ. 1952. - - Uber eine Verlandungsfolge weichsel-interstadialen Alters in Harksheide bei Hamburg. - Eiszeitalter u. Gegenwart 6, S. 116-124, Ơhringen/ Württ. 1955.

Jessen, K. \& MiLthens, V.: Stratigraphical and Paleontological Studies of Interglacial Freshwater Deposits in Jutland and Northwest Germany. - Danmarks Geol. Unders. 2. R. 48, 379 S., Kopenhagen 1928.

Koert, W.: Erläuterungen zur Geologischen Karte von Preußen, Lieferung 176, Blatt Bergedorf. 79 S., Berlin 1912.

LüDI, W.: Die Pflanzenwelt des Eiszeitalters im nördlichen Vorland der Schweizeralpen. - Veröff. geobot. Inst. Rübel 27, 208 S., Zürich 1953.

Lüttig, G. \& Rein, U.: Das Cromer-(Günz/Mindel-)Interglazial von Bilshausen (Untereichsfeld). Geol. Jb. 70, S. 159-166, Hannover 1954.

Range, P.: Neue pflanzenführende Ablagerungen aus Südost-Holstein. - Unveröffentl. Mskr. im Geolog. Landesamt Schleswig-Holstein, Kiel 1945.

REICH, H.: Die Vegetationsentwicklung der Interglaziale von Großweil-Ohlstadt und Pfefferbichl im Bayerischen Alpenvorland. - Flora 140, S. 386-443, Jena 1953.

Selle, W.: Das Interglazial von Praschnitz. - Geol. Jb. 77, S. 319-328, Hannover 1960.

Środoñ, A.: Interglacial in Szelag near Poznań. - Biul. Instyt. Geolog. 100, S. 229-316, Warschau 1956.

Weber, C. A.: Uber die diluviale Flora von Fahrenkrug in Holstein. - Beibl. Botan. Jb. Nr. 43, 18, S. 1-13, Leipzig 1893.

Woldstedt, P.: Norddeutschland und angrenzende Gebiete im Eiszeitalter, 2. Aufl., 467 S., Stuttgart 1955.

ZAGwiJn, W. H.: Vegetation, Climate and Radiocarbon Datings in the Late Pleistocene of the Netherlands. Part I: Eemian and Early Weichselian. - Memoirs Geolog. Found. Netherlands N.S. 14, S. 15-46, 1961.

Manuskr. eingeg. 30. 11. 1961.

Anschrift des Verfassers: Dr. F.-R. Averdieck, Geologisches Landesamt Schleswig-Holstein, Kiel, Mecklenburger Straße 22/24. 